Ciencias $\approx$ Agua

DOI: $10.24850 / j-$ tyca-2020-06-04

Artículos

\title{
Entre una GIRH inconsistente e inseguridades hídricas multiformes: la cuenca transfronteriza del río Hondo en la política mexicana
}

\section{Between an erratic IWRM and multifaceted water insecurities: the transboundary Hondo River Basin in Mexican policy}

Edith Kauffer ${ }^{1}$, ORCID: https://orcid.org/0000-0003-4238-9782

${ }^{1}$ Centro de Investigaciones y Estudios Superiores en Antropología Social (CIESAS)-Sureste, San Cristóbal de Las Casas, México, ekauffer@ciesas.edu.mx

Autora para correspondencia: Edith Kauffer, kauffer69@hotmail.com

\section{Resumen}

La gestión integrada de recursos hídricos (GIRH) y la seguridad hídrica (SH) constituyen dos componentes definidos en el ámbito internacional y están aceptados como pilares de la política hídrica mexicana. Resulta pertinente para la investigación socio-política analizar su concreción en estudios de caso locales, en particular, en aquellos donde existen 
estructuras de gestión creadas explícitamente en el marco de la GIRH. Es el caso de la cuenca del río Hondo, localizada al extremo sur de la república mexicana, compartida con Belice y Guatemala. En dicho escenario, este artículo busca analizar la implementación de estos dos principios internacionales, a partir de las perspectivas de los actores involucrados en la Comisión de Cuenca del Río Hondo (CCRH), formada en la parte mexicana. Se fundamenta en un trabajo de campo que duró varios años en la cuenca y consistió en la realización de entrevistas con distintos actores en la observación de procesos vinculados con las instituciones creadas en materia de GIRH, y en documentos de la política hídrica mexicana. Después de presentar los acercamientos teóricos y los debates en materia de GIRH y SH a escala internacional y su traducción en la política hídrica mexicana, este texto evidencia una GIRH inconsistente en la porción mexicana de la cuenca del río Hondo, un gran desconocimiento de la noción de seguridad hídrica, en paralelo con el surgimiento de una serie de inseguridades hídricas multiformes susceptibles de generar acciones locales de $\mathrm{SH}$, encaminadas hacia la resolución de problemas agudos.

Palabras clave: cuenca del río Hondo, GIRH, seguridad hídrica, comisión de cuenca, política hídrica.

\section{Abstract}

Integrated Water Resources Management (IWRM) and Water Security (WS) are both defined components at international scale imported into Mexican water policy as essential pillars. Social and political research proposes to analyze relevant local case studies, especially when IWRM institutions have been created for the purpose of its implementation. 
Located at the South of Mexico, the Hondo river basin, which is a transboundary watershed shared with Belize and Guatemala constitutes an adequate example to deepen this study. The paper analyzes how both international water principle of IWRM and WS are implemented, from local stakeholders' perspectives, in the Mexican part of the Hondo river basin, through its recent created Basin Commission. It is based on an extended fieldwork along several years that consisted in interviews, IWRM policy ethnography and Mexican water policy written sources. The article deals first with theoretical and current debates in international literature on IMRM and WS and their Mexican version to then reveal the existence of an erratic IWRM in the Mexican part of the Hondo river basin as well as a lack of knowledge about the water security concept that coexists in parallel on the uprising of a variety of multifaceted waterinsecurities, that are nevertheless prone to generate local actions to resolve acute problems through WS.

Keywords: Hondo River Basin, IWRM, water security, basin commission, water policy.

Recibido: 07/10/2019

Aceptado: 05/03/2020

\section{Introducción}


En los confines del sur de México se encuentra la cuenca transfronteriza del río Hondo, cuya característica es ser trinacional, es decir, compartida con Guatemala, ubicada en la parte alta, y con Belice, país que colinda con México en la parte media y baja de este territorio.

Como otras cuencas transfronterizas de México con sus vecinos del sur, cuyo número varía según las fuentes (Conagua, 2016; García \& Kauffer, 2011), debido a la ausencia de delimitaciones establecidas por y entre los Estados nación, tal como sucede en el caso del río Suchiate (Kauffer, 2017) o del Usumacinta (Benítez, 2010; CCGSS (2015), la delimitación de la cuenca del río Hondo presenta discrepancias en la literatura (Benítez, 2010; Olvera, Kauffer, Schmook, \& Huicochea, 2011) y entre los actores involucrados en su gestión (Conagua, 2007).

Como resultado de varios años de trabajo en la elaboración de mapas de cuencas transfronterizas en la región, esta cuenca tiene un espacio con una extensión de $14859 \mathrm{~km}^{2}$, donde 33.9\% se encuentra en Guatemala; $33.4 \%$ en Belice y $32.8 \%$ en México, es decir, una cuenca transfronteriza distribuida de forma equilibrada en superficie entre los tres países.

Según datos del 2010, la cuenca trinacional ocupa la parte noroeste del departamento del Petén en Guatemala, donde nace el Arroyo Azul, tributario del Hondo: se extiende en parte de los municipios de San José, Flores y Melchor de Mencos. En Belice se localiza en los distritos de Corozal y Orange Walk. En México, está al sur de los estados de Campeche y Quintana Roo, respectivamente, en los municipios de Calakmul y de Othón P. Blanco. 
La cuenca del río Hondo contaba con 88145 habitantes en el 2010, de los cuales 48020 se encontraban en Belice; sólo 4350 en Guatemala, y 35775 en México. Así, de un total de 213 localidades, 115 eran mexicanas, 78 ubicadas en Belice y 20 en Guatemala. La densidad poblacional promedio de la cuenca del río Hondo era de 6 habitantes $/ \mathrm{km}^{2}$, en una distribución poco uniforme: la parte guatemalteca, escasamente habitada con pequeñas localidades dispersas; la porción beliceña, con localidades más pobladas; y la parte mexicana, con asentamientos de diversos tamaños, como la ciudad de Chetumal, la más dinámica de la cuenca y localidades de distintos tamaños, cuyo número elevado contrastaba con sus vecinos. En materia de vegetación, $66 \%$ de la cuenca presentaba una cobertura de selvas, aunque muy diferenciada por país: $78.8 \%$ de la porción de Guatemala eran selvas; $73.8 \%$ de la fracción mexicana de la cuenca poseía cobertura vegetal, y por último, sólo $45.3 \%$ de Belice, donde, sin embargo, se registraba una situación extrema de deforestación en el distrito de Corozal, ubicado en la parte baja, con sólo $4.87 \%$ de cobertura de selvas, a causa de las grandes extensiones agrícolas dedicadas a los cultivos de caña de azúcar (Figura 1). 


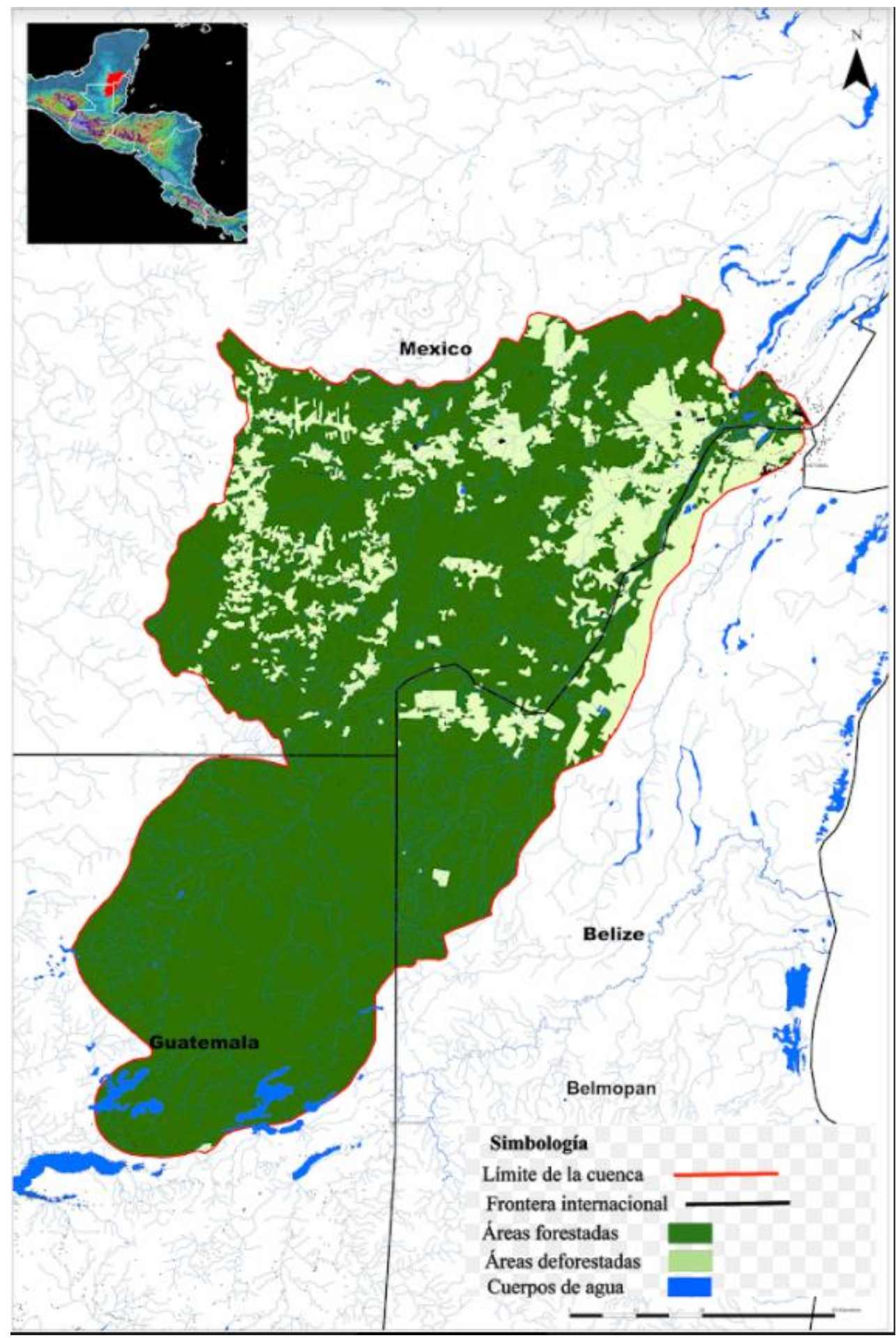

Figura 1. Cobertura vegetal en la cuenca transfronteriza del río Hondo. 
La cobertura vegetal (o su ausencia) se relaciona de manera directa con la presencia de 15 áreas protegidas (AP), de las cuales, 10 se localizan en Guatemala: entre ellas, las distintas zonificaciones de la Reserva de la Biósfera Maya, es decir, un área inicialmente despoblada donde el avance de la frontera agrícola y de los asentamientos humanos han sido importante desde el año 2010. Sin embargo, no existe un dato reciente y preciso acerca de este fenómeno, debido a que el Censo de Población de Guatemala aún no publica resultados desglosados del año 2019 y ha sido cuestionado por instituciones académicas. México, por su parte, cuenta con tres áreas naturales protegidas (ANP) en la cuenca del río Hondo, que explican cierta preservación de la cobertura vegetal, todas ubicadas en el estado de Campeche, donde destaca la Reserva de la Biósfera de Calakmul. En la parte alta de la cuenca, Belice posee una pequeña porción con dos AP de extensión limitada, en comparación con las extensas áreas de sus vecinos. Cabe destacar que la denominación de áreas naturales protegidas (ANP) es propiamente mexicana, por lo que la referencia para Guatemala y Belice son las áreas protegidas (AP).

Al igual que los otros países de la Organización para la Cooperación y el Desarrollo Económico (OCDE), México ha adoptado los principios internacionales de política hídrica vigente a escala internacional, dos de ellos son la gestión integrada de los recursos hídricos (GIRH), que orienta un modelo de política desde los años 1990; y la seguridad hídrica (SH), cuya aparición es más reciente, a escala internacional, y fue acuñada en el año 2000.

Con base en el contexto antes descrito, este artículo propone responder a las siguientes preguntas: ¿cómo se traducen los principios internacionales de la gestión integrada de recursos hídricos (GIRH) y de 


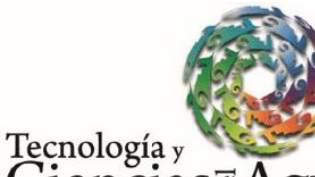

Ciencias $\stackrel{\unlhd}{\varpi}$ Aua
2020, Instituto Mexicano de Tecnología del Agua Open Access bajo la licencia CC BY-NC-SA 4.0

(https://creativecommons.org/licenses/by-nc-sa/4.0/)

la seguridad hídrica (SH) en la cuenca transfronteriza, a partir de las perspectivas de los actores involucrados en la Comisión de Cuenca del Río Hondo (CCRH)? ¿Cuáles son las relaciones detectadas entre la GIRH y la $\mathrm{SH}$ en la cuenca del río Hondo, en tanto al escenario abordado desde el caso mexicano?

El estudio de caso refiere a la cuenca del río Hondo antes descrita y aunque considera la cuenca en su conjunto, se centra en la perspectiva de la GIRH y de la SH en México. En la medida en que las políticas hídricas se encuentran ancladas en las fronteras políticas, las primeras son definidas por los gobiernos nacionales $y$, por lo tanto, presentan diferencias sustanciales entre los tres países (Kauffer, 2018; Kauffer \& Mejía, 2020), con historias y marcos institucionales propios, por lo que no resulta posible desarrollar en este trabajo un análisis más allá de México. En efecto, se trata del único Estado nación con una estructura institucional relacionada con la GIRH en la cuenca estudiada.

Como evidencia tomada de otras publicaciones (Kauffer, 2018; Kauffer \& Mejía, 2020) sobre cuencas transfronterizas de la región, varias razones conllevaron a tomar la decisión de enfocar el análisis del río Hondo en la parte mexicana. En primer lugar, aunque la extensión física de la cuenca del río Hondo corresponde a tres Estados nación, éstos se caracterizan por tres políticas hídricas que, aunque reivindican a la GIRH en su centro, presentan realidades institucionales muy contrastadas. Aunado a ello, no existe, hasta la fecha, colaboración con Guatemala en materia transfronteriza y sólo algunas relaciones de tipo informal con Belice, además de algunas intenciones de cooperación que no se reflejan en las actividades de la CCRH. 
Así, después de analizar el contexto institucional mexicano sobre lo transfronterizo en publicaciones previas (Kauffer, 2018), al igual que la problemática histórica y actual de Belice en materia de política hídrica y de GIRH (Kauffer \& Mejía, 2020), este trabajo expondrá en las siguientes páginas un estudio de caso transfronterizo específico, visto en sus relaciones con la GIRH y la SH. De esta manera, la decisión de centrar el trabajo sólo en la parte mexicana no excluye la posibilidad de contribuir de modo académico a una mirada transfronteriza en un territorio no construido como tal por los actores involucrados en las políticas hídricas, y necesariamente requiere entender cómo se concretan la GIRH y la SH, al igual que cuáles son sus relaciones en cada contexto político nacional en las distintas escalas.

Este artículo abordará sucesivamente los acercamientos teóricos en materia de $\mathrm{GIRH}$ y de $\mathrm{SH}$, antes de presentar los materiales y métodos que determinan el contexto de una investigación que se inscribe en el mediano plazo para presentar los resultados organizados en torno a dos grandes ejes de análisis propuestos para la discusión: las inconsistencias de la GIRH en la porción mexicana de la cuenca del río Hondo, a partir de un análisis de su principal instrumento; y las relaciones entre ambos pilares de la política del agua en México expresadas en tres modalidades: 1) una "GIRH" caracterizada por una serie de inconsistencias y sin vínculo con la $\mathrm{SH}$; pero 2) que coexiste con inseguridades hídricas multiformes, y 3) el surgimiento de acciones generadas en el contexto de la GIRH tendientes a la $\mathrm{SH}$ en condiciones críticas. 


\section{Gestión integrada de recursos hídricos (GIRH) y seguridad hídrica (SH): acercamientos teóricos}

¿Qué se entiende por GIRH y SH? El siguiente apartado aborda las dos nociones, las distintas visiones en torno a ellas y los cuestionamientos de la literatura internacional más reciente.

\section{La GIRH y sus cuestionamientos}

La gestión integrada de los recursos hídricos (GIRH) es hoy en día el eje principal de la política hídrica en el mundo y en México. Según el Global Water Parternship (GWP, 2000a), la gestión integrada de los recursos hídricos (GIRH) es: "Un proceso que promueve el manejo y desarrollo coordinado del agua, la tierra y los recursos relacionados, con el fin de maximizar el bienestar social y económico resultante de manera equitativa sin comprometer la sustentabilidad de los ecosistemas vitales".

En el centro de este planteamiento está la integración de diferentes fenómenos y actores que entran en juego en la gestión del agua (GWP, 2000a). Hoy en día, es comúnmente aceptado en las esferas políticas y académicas que la GIRH constituye una herramienta clave encaminada 
hacia la sustentabilidad del agua y ofrece un "enfoque comprehensivo y holístico" (Biswas, 2004), además de la promesa de un futuro mejor en la gestión del agua.

En fechas recientes, el Handbook for integrated water resources management in transboundary basins of rivers, lakes and aquifers (INBO et al., 2012) presenta una síntesis de la GIRH y establece que ésta depende de tres pilares para su implementación: 1) los instrumentos de gestión que incluyen la evaluación de los recursos hídricos, el intercambio de información, los instrumentos socio-económicos y regulatorios, de la misma manera que los planes de GIRH; 2) un ambiente propiciador que incorpora políticas públicas, un marco legal, estructuras financieras y de incentivos; 3) los roles institucionales que se centran en la relación centro-local y público-privado, a escala de la cuenca, así como en la construcción de capacidades.

Un elemento central de la propuesta de la GIRH reside en la cuenca como unidad de gestión y escenario de concreción de acciones. Actualmente, dicho planteamiento constituye el principal lineamiento internacional de las intervenciones de política hídrica. Sin embargo, la noción de cuenca presenta dos grandes aproximaciones básicas: la físicotécnica y la sociopolítica, siendo la primera predominante en la literatura científica y en la implementación de la política hídrica mexicana.

A pesar de la adopción de la GIRH, ésta sólo ha sido una ruptura discursiva en la política hidráulica mexicana, tal como lo han evidenciado diversos estudios de caso (Kauffer, 2014; Kauffer \& Gallardo, 2019a; Maganda, 2019; Mejía \& Pliego, 2019).

Hasta la fecha, la GIRH ha adoptado dos modalidades: el manejo integral de cuencas y la gestión integrada de los recursos hídricos. El 


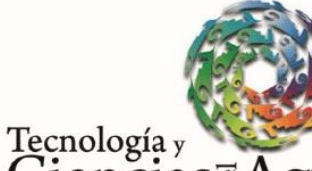

Ciencias $₫$ Agua
2020, Instituto Mexicano de Tecnología del Agua Open Access bajo la licencia CC BY-NC-SA 4.0

(https://creativecommons.org/licenses/by-nc-sa/4.0/)

primero centrado históricamente en los aspectos técnicos hidráulicos, y después, en el manejo de suelos y de los recursos forestales. La segunda corresponde a la definición propuesta en este artículo y ubica los aspectos sociales y políticos en su centro. Así, tomar en cuenta los aspectos políticos, institucionales y económicos es esencial, y no reducirse a componentes técnicos, hidrológicos, ingenieriles o ambientales. Es decir, que se refiere a una amplia perspectiva de la gestión integrada de recursos hídricos.

En el mundo, y en particular en México, las políticas implementadas a escala de cuencas han seguido la visión técnica (principalmente hidráulica) predominante. Esta perspectiva se relaciona con el anclaje de la GIRH en una definición de las cuencas como unidades naturales, visión que tiende a minimizar su dimensión social y política, y se traduce en una intocabilidad de la dimensión "natural" de una cuenca, y en una dificultad para abrir el debate crítico en torno al concepto de cuenca y a la GIRH, tal como lo han hecho en los últimos años reconocidos científicos sociales en el ámbito internacional (Biswas, 2004; Biswas, 2008; Molle, 2006; Warner, Wester, \& Bolding, 2008). De esta visión se deriva la definición tradicional de la cuenca como el área de la superficie terrestre delimitada por un parteaguas (línea que une los puntos de mayor altura), por donde el agua de lluvia escurre y tiende a ser drenada a través de una red de corrientes que fluyen hacia una corriente principal, y por ésta hacia un punto común de salida, centrada en los aspectos físicos e hidrográficos.

Desde una visión crítica, la noción de cuenca debe tomar en cuenta los aspectos sociales y considerar que las cuencas no son espacios definidos con base en criterios naturales, es decir, que no se trata de espacios explicados a priori por la naturaleza (Wester \& Warner, 2002) 
sino territorios apropiados. Desnaturalizar las cuencas es una necesidad $y$, sin duda, un primer paso para concretar una verdadera GIRH, porque invita a observar a las cuencas en su totalidad, paso previo para el diseño de acciones integrales e integrativas.

El enfoque de cuencas adoptado por el gobierno mexicano a lo largo del siglo XX es indisociable de los aportes de la investigación desarrollada a escala internacional y de su adopción por la comunidad científica mexicana. Cabe subrayar que la predominancia de la hidráulica a lo largo del desarrollo de la visión de cuencas y su concreción mediante las intervenciones del Estado constituyó, en su momento, un gran avance que enfrenta hoy en día el reto de incorporar a la GIRH otras disciplinas, como las ciencias sociales, principalmente.

A través de la Ley de Aguas Nacionales de 1992 se adoptó la GIRH como el eje de la política hídrica mexicana, cuando la noción se convirtió en un principio internacional. Ésta se reforzó mediante las reformas a la ley en el año 2004 y finalmente se concretó en México por medio de una doble estructura: por un lado, las organizaciones de cuenca que corresponden a los consejos, comisiones y comités susceptibles de integrar a los actores sociales en distintas escalas de cuencas que fueron creados desde la mitad de la década 1990, y principalmente en los albores del siglo, como el Consejo de Cuenca de la Península de Yucatán (CCPY) en 1999 y, por el otro, los organismos de cuenca, como parte de la estructura gubernamental desde 2004.

\section{La SH desde perspectivas internacionales y en México}


En la literatura internacional, el concepto de seguridad hídrica ( $\mathrm{SH}$ ) aparece en los lineamientos de las políticas hídricas, y es objeto de debate entre organizaciones internacionales y agencias financiadoras. Su uso por la literatura académica, en donde las definiciones del término y sus escalas de aplicación varían según las disciplinas del conocimiento científico (Cook \& Bakker, 2012), ha aumentado en frecuencia. En su revisión, Cook Bakker (2012) evidencian cuatro grandes temáticas que se entrecruzan con los debates en materia de política del agua: 1) la seguridad hídrica asociada con indicadores tradicionales de escasez y de estrés hídrico; 2) la vulnerabilidad relacionada con sequías e inundaciones; 3) la seguridad enfocada hacia las dimensiones humanas, en particular en materia de seguridad alimentaria en los últimos años, y 4) la SH relacionada con la sustentabilidad.

Según la Global Water Partnership (GWP, 2000b): "La seguridad hídrica en cualquier nivel, desde la esfera doméstica hasta lo global significa que cada persona tiene acceso a suficiente agua segura a un costo alcanzable para llevar una vida limpia, sana y productiva, mientras asegura que el entorno natural está protegido y valorizado". En la opinión de Cook y Bakker (2012) esta definición constituye el ejemplo de una visión amplia que integra distintos componentes. Estos autores mencionan distintos enfoques en materia de $\mathrm{SH}$, que van desde los más acotados hasta los más amplios, y argumentan la necesidad de una perspectiva integradora de la noción. En efecto, la diversidad de factores que interviene en la gestión de los recursos hídricos implica una 


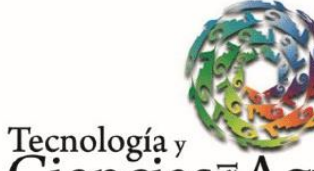

Ciencias đAgua
2020, Instituto Mexicano de Tecnología del Agua Open Access bajo la licencia CC BY-NC-SA 4.0

(https://creativecommons.org/licenses/by-nc-sa/4.0/)

perspectiva que concilie diversos métodos, y trabaje en una noción integradora de este concepto aún emergente (Cook \& Bakker, 2012).

Parte del debate sobre la SH que mencionan Cook y Bakker (2012) es su relación con la GIRH. Por un lado, la SH presenta ciertos componentes comunes y paralelos con la GIRH, e incluso enfrenta los mismos desafíos en materia de imprecisiones y de generalidades teóricas, y entonces resulta "atractiva" por estas mismas razones. En la opinión de estos autores, ambos conceptos deben considerarse como complementarios. En consecuencia, las acciones de GIRH y de SH también deberían ser complementarias en su implementación.

En la actualidad, el debate se orienta entre la definición cuantitativa de la SH y algunas propuestas más amplias que subrayan el carácter limitado de un enfoque centrado en indicadores. En efecto, en muchas de las distintas propuestas, como aquella de la Academia Mexicana de Ingenieros (Martínez, Díaz-Delgado, \& Moeller, 2017), y en parte de la literatura internacional (Norman, Dunn, Bakker, Allen, \& De Albuquerque, 2013), los autores retoman indicadores tradicionales de disponibilidad, de grado de presión sobre el recurso, de cobertura de agua entubada y de alcantarillado, o de calidad del agua, los cuales se convierten en mediciones de la SH y, a la postre, consisten sólo en reutilizarindicadores tradicionales para evocar la seguridad hídrica.

En México, la $\mathrm{SH}$ se define, a partir de la propuesta de la Organización de Naciones Unidas (ONU), como: "La capacidad de la población de salvaguardar el acceso sostenible a cantidades adecuadas y de calidad aceptable de agua para sostener los medios de sustento, el bienestar humano y el desarrollo socioeconómico, para garantizar la protección contra la contaminación del agua, y los desastres relacionados 
con el agua, y para preservar los ecosistemas en un clima de paz y estabilidad política" (Conagua, 2014). Se trata de una visión cercana a los cuatro componentes antes mencionados por Cook y Bakker (2012), así como por el concepto del GWP en el año 2000 (GWP, 2000b).

Sin embargo, la noción no figura en la Ley de Aguas Nacionales (LAN), pues se trata de un concepto que aparece en el año 2000, en fechas posteriores a la GIRH y a la propia ley. El concepto de seguridad asociada con el agua que predomina históricamente en México presenta distintos adjetivos; los 19 documentos oficiales correspondientes a un periodo de 1992 a 2016, revisados por Kauffer y Gallardo (2019), permiten distinguir la noción de seguridad hidráulica en 11 de éstos, seguida por la seguridad del suministro en nueve de los casos y por la seguridad nacional en ocho de ellos. La seguridad hidráulica es la más antigua -aparece desde 1992 - , remite a las obras hidráulicas como las presas y sigue vigente. Las seguridades de suministro y seguridad nacional son acuñadas, respectivamente, en 2002 y 2003: la primera refiere a la dotación en servicios de agua a la población, y la segunda está presente en el discurso de la política del agua entre 2003 y 2007; y desaparece y vuelve a aparecer en el Programa Nacional Hídrico 20142018. Los documentos oficiales también mencionan que las nociones de seguridad social y pública únicamente en 2 o 3 ocasiones. Por último, a la par del concepto de seguridad hídrica, las nociones de seguridad alimentaria y jurídica, que revelan la inserción del concepto en las discusiones internacionales, han sido utilizadas desde el año 2014.

Así, el concepto de seguridad hídrica en México aparece de forma tardía en el discurso de la política hídrica y bajo la modalidad de distintos tipos de seguridades vinculadas con el agua, entre las cuales predominan 


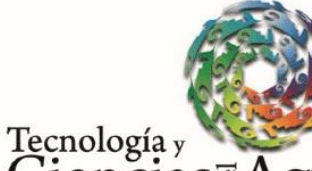

Ciencias $₫$ Agua
2020, Instituto Mexicano de Tecnología del Agua Open Access bajo la licencia CC BY-NC-SA 4.0

(https://creativecommons.org/licenses/by-nc-sa/4.0/)

aquellas que refieren a las dimensiones hidráulicas y de obras ingenieriles a la seguridad nacional y, de manera más reciente, a distintas seguridades "adjetivadas", que tienden a incorporar algunos aspectos relacionados con las necesidades de la población, como las desigualdades.

Es hasta el Programa Nacional Hídrico 2014-2018, que la noción de seguridad hídrica se precisa y asocia con 21 indicadores, así como con un índice global de seguridad hídrica (IGSH) (Conagua, 2014), que presentan una serie de deficiencias y contradicciones, además de limitadas posibilidades de aplicación en distintas escalas de cuencas (Kauffer \& Gallardo, 2019), es decir, que difícilmente permiten una articulación con el otro pilar de la política hídrica: la gestión integrada de recursos hídricos.

Los indicadores cuantitativos, sean los tradicionales (utilizados para evaluar distintos aspectos de los recursos hídricos o los nuevos construidos a propósito para la $\mathrm{SH}$ ), como el ISGH propuesto por el $\mathrm{PNH}$ 2014-2018, plantean la viabilidad de esta perspectiva predominante en los debates internacionales de políticas hídricas y de la literatura focalizada en la medición de este principio, así como de su novedad cuando se toma en cuenta el uso recurrente de indicadores tradicionales.

En definitiva, desde las ciencias sociales y políticas existe un planteamiento alternativo que propone la siguiente perspectiva de $\mathrm{SH}$ : "La seguridad hídrica para un grupo de actores implica comúnmente la inseguridad hídrica para otros. En este sentido, la seguridad hídrica es a la vez, un reflejo histórico y una fuerza importante que traduce relaciones de poder predominantes. En el contexto actual de las presiones sobre los recursos hídricos, la inseguridad hídrica es una relación sociopolítica que es sentida de una manera excesivamente fuerte por los grupos sociales 


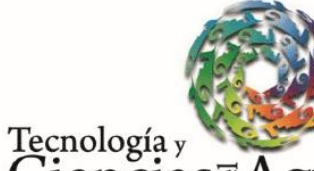

Ciencias $₫$ Agua
2020, Instituto Mexicano de Tecnología del Agua Open Access bajo la licencia CC BY-NC-SA 4.0

(https://creativecommons.org/licenses/by-nc-sa/4.0/)

menos poderosos en el ámbito socioeconómico y político" (Hidalgo, Boelens, \& Vos, 2017).

Desde este punto de vista, la SH refleja y traduce las relaciones de poder en un escenario en materia hídrica. El agua no es neutral y no se reduce a flujos, a fenómenos climáticos o ambientales, sino que presenta dimensiones políticas y está anclada en escenarios sociales ubicados en un espacio y tiempo determinados (Kauffer, 2019). En consecuencia, si es posible sólo medir la SH como la primera perspectiva lo pretende (es decir, a partir de indicadores cuantitativos), es recomendable, siguiendo la definición propuesta, propiciar la identificación de nudos de inseguridad hídrica. Seguridad(es) e inseguridad(es) hídricas están estrechamente vinculadas en esta perspectiva comprensiva del agua. Esta propuesta lleva a incluir en el análisis la existencia de conflictos con diversos orígenes, como las actividades relacionadas con la exploración y explotación de pozos petroleros; la construcción de hidroeléctricas; la extracción de sedimentos en ríos, como resultado de relaciones de poder entre distintos actores. De la misma forma, las tensiones y disputas en torno al agua entre el uso doméstico y la agricultura, en particular los monocultivos de agroexportación; el ecoturismo; la ganadería; pero también en la construcción de obras hidráulicas y de control, en contra de las inundaciones, y finalmente, la calidad y contaminación del agua, componen elementos que suelen expresar distintas relaciones de poder $y$, por ende, se convierten en seguridades-inseguridades hídricas según sean vividas y experimentadas por los distintos actores sociales en función de sus diferentes actividades y posiciones socio-políticas. Esta visión resulta muy pertinente para profundizar la situación de la cuenca del río Hondo en la materia, y permite ir más allá de los datos 
cuantitativos, que no están disponibles a escala de la cuenca en México, ni en Belice o en Guatemala.

\section{Materiales y métodos}

Este trabajo es el resultado de más de una década de seguimiento de las problemáticas del río Hondo desde el año 2003, del cual se han derivado análisis con perspectivas distintas a la presentada en este artículo, pero de cuyas experiencias se alimenta.

Durante la última década, la investigación abarcó tres grandes periodos. En marzo de 2014 se realizaron recorridos en la parte mexicana de la cuenca del río Hondo; en Belice, en noviembre de 2014, se trabajó en la capital de aquel país, Belmopán, para identificar distintos actores sociales relacionados con la cuenca y sus diversas problemáticas. En ambos casos, se efectuaron entrevistas semiestructuradas con 15 informantes clave en México y 11 en Belice.

Específicamente, la reflexión que se desarrolla a continuación se realizó en el marco de un proyecto financiado por la Convocatoria de Problemas Nacionales del Consejo Nacional de Ciencia y Tecnología 2014, integrado por ocho estudios de caso distribuidos a lo largo de la geografía nacional, el cual propone analizar dos pilares de la política hídrica 
mexicana (GIRH y $\mathrm{SH}$ ) desde las experiencias locales. El río Hondo y su Comisión de Cuenca formaron parte de uno de los ocho estudios de caso.

El trabajo de campo de este caso específico consistió en dar seguimiento a las actividades de la Comisión de Cuenca del Río Hondo $(\mathrm{CCRH})$ y a sus labores, en la medida en que se trata del principal mecanismo que expresa la GIRH en la cuenca estudiada. En este tercer periodo de estudio de la cuenca se efectuaron entrevistas semiestructuradas con 13 de los actores clave más involucrados en las labores de la CCRH y pertenecientes al gobierno federal, estatal, al mundo académico y a organizaciones de base que forman parte de la Comisión de Cuenca, y están relacionados con actividades llevadas a cabo en este escenario. Esto se decidió después de analizar en los dos periodos previos las redes de actores sociales existentes y sus relaciones en la cuenca. Además, la fuente de financiamiento obligó a que la investigación se centrara en la parte mexicana de la cuenca del río Hondo; por ello, no se trabajó directamente en torno a la dimensión transfronteriza y tampoco en Belice, como en proyectos anteriores, aunque, en materia de $\mathrm{GIRH}$, es indudablemente relevante y ésta debe ser considerada para futuros análisis. El trabajo también se sustenta con la observación etnográfica de reuniones y eventos llevados a cabo en la región desde el año 2003, donde confluyeron distintos actores relacionados con esta cuenca, en particular la CCRH y con las políticas hídricas de los tres países.

\section{Resultados y discusión}


Los resultados del estudio de caso del río Hondo evidencian una GIRH marcada por una serie de inconsistencias si se observa la labor de la Comisión de Cuenca del Río Hondo (CCRH). Por su parte, las relaciones entre GIRH y SH presentan distintas combinaciones, a partir de una ausencia de relación en la práctica entre ambos pilares.

\section{La cuenca del río Hondo: escenario de una GIRH inconsistente}

Una serie de realidades históricas que han marcado la conformación y el funcionamiento de la Comisión de Cuenca del Río Hondo (CCRH), sumadas a un proceso resultante de la transformación de la política mexicana permiten observar una GIRH inconsistente en el río Hondo a lo largo de los últimos años.

\section{Inconsistencias originarias de la GIRH: la CCRH}




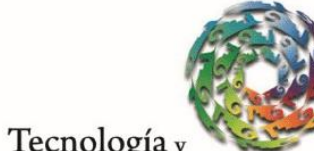

Ciencias $₫$ Agua
2020, Instituto Mexicano de Tecnología del Agua Open Access bajo la licencia CC BY-NC-SA 4.0

(https://creativecommons.org/licenses/by-nc-sa/4.0/)

Como parte de la estructura de la GIRH que el gobierno federal organizó en todo el país de forma top-down, es decir, a partir de un modelo diseñado desde la Ciudad de México y replicado en un primer momento en 25 grandes consejos de cuenca, y a continuación en 26, fue creado el Consejo de Cuenca de la Península de Yucatán (CCPY), inaugurado por la Comisión Nacional del Agua (Conagua) en 1999. Los Consejos de Cuenca se instituyeron entre el año 1993 y 2009; pero si se excluye el primero, en la cuenca Lerma-Chapala en el año 1993 y el último de la Costa Pacífico Centro en 2009, la época de creación de estas organizaciones se extiende a lo largo de cinco años, de 1996 a 2000, y en particular se concentra en dos años. Así, entre 1999 y 2000 se establecieron 22 de los consejos de cuenca, y justamente el CCPY se inscribió con esta ola de formación.

En un primer momento, muchos de los consejos de cuenca funcionaron a través de grupos de trabajo relacionados con las distintas problemáticas planteadas en el seno de una gran cuenca $y$, en muchos casos, fueron emanados de la propia Conagua. Sin embargo, algunos ejemplos, como el Consejo de Cuenca de la Costa de Oaxaca, que inauguró el primer comité de cuenca a escala nacional en el río de Los Perros en 1999; Ios consejos de cuenca de los ríos Grijalva-Usumacinta y de la costa de Chiapas, caracterizados por la creación de varios comités desde 2003, fueron reemplazados paulatinamente por los llamados órganos auxiliadores, es decir, las comisiones a escala de subcuencas, y los comités de cuenca, para desarrollar acciones de GIRH en las microcuencas.

EI CCPY siguió organizando reuniones alrededor de los grupos de trabajo especializados (GTES), a diferencia de otros consejos, que sustituyeron estos grupos por los órganos auxiliares. En tal contexto, el 
primer órgano auxiliar creado fue la Comisión de Cuenca del Río Hondo (CCRH) en 2009, 10 años después de su inauguración como Consejo de Cuenca. A la fecha, en el CCPY, las comisiones y los comités de cuenca siguen trabajando en paralelo con los GTES, de tal forma que la organización espacial del CCPY es posterior a su estructura temática y ambas coexisten.

La CCRH se inauguró en 2009, después del diagnóstico de la cuenca en 2007. El documento titulado Diagnóstico para el Manejo Sustentable de la Cuenca Internacional del Río Hondo al 2025 fue financiado por la Conagua (2007), como resultado de un acuerdo tomado en 2003, en el seno de la Comisión Internacional de Límites y Aguas México-Belize (sic) (CILA); es decir, como una colaboración binacional, aunque bajo liderazgo mexicano, proceso que no estuvo exento de tensiones:

"¿Por qué no aprobamos el acuerdo? En el momento que lo aprobamos, en enero de 2005, ellos lo aprobaron en el mismo mes, el mismo mes, enero de 2005, coincidentemente con una Alta Reunión de la Comisión Binacional México-Belize, que se celebró en Belmopan, Belice, en enero de 2005. Firmó el Acta el secretario de Relaciones Exteriores, el secretario Derbez, con el primer Ministro de Belice, en la cual se dice que los gobiernos anunciaron su aprobación formal al Acta 12 de la Comisión Internacional de Límites y Aguas para que el estudio denominado 'Diagnóstico para el Manejo Sustentable del Agua en la Cuenca Internacional del río Hondo México-Belice al año 2025', así se denomina el estudio, mismo que iniciará durante el 2005 y será financiado por el gobierno de México a través de 
la Comisión Nacional del Agua, en coordinación con el Servicio Nacional de Meteorológico de Belice; tuvo hasta el anuncio de los cancilleres, que esto refleja la importancia que tienen para Belice y para México el que se hiciera este estudio". Funcionario del gobierno federal mexicano, Segunda Reunión del Consejo de Cuenca de la Península de Yucatán, Grupo de Trabajo Especializado en Saneamiento de Quintana Roo (GTESQROO), 24 de agosto de 2006.

El diagnóstico excluyó desde un inicio a Guatemala. El trabajo de campo realizado en la región desde el año 2003 evidenció dos situaciones históricas totalmente encontradas que explican cómo esta cuenca trinacional se convirtió en cuenca binacional desde el punto de vista político. En primer lugar, existe un marcado desinterés guatemalteco para una cuenca ubicada en los confines de su territorio, en un área casi despoblada y de difícil acceso, como lo subrayaba un entrevistado:

"Primero es que la cuenca del río Hondo... éstas son áreas prácticamente inexploradas en el país, aquí no vas a encontrar una mayor colaboración institucional". Miembro de organización no gubernamental, ciudad de Guatemala, 29 de mayo 2007.

En segundo lugar, es patente la situación conflictiva de la llamada "línea de adyacencia" entre Belice y Guatemala, que ha sido llevada a un juicio fronterizo ante la Corte Internacional de Justicia de la Haya, por desacuerdos en materia de delimitación y ante la imposibilidad de dirimir 
el diferendo por otras vías (Alija, 2019). El siguiente extracto del correo electrónico enviado por el viceministro de Relaciones Exteriores de Guatemala es extremadamente claro al respecto, y el cual menciona que:

"Agradezco por copiarme este correo y deseo aclararle que Guatemala NO tiene fronteras definidas aun con Belice, lo cual sera sometido a la Corte Internacional de Justicia, por lo que mucho apreciaré se corrija el lenguaje utilizado y, en todo caso, se refieran a la "Linea de Adyacencia entre Guatemala y Belice", lenguaje acordado por ambos paises" (sic). Correo recibido el 19 de marzo de 2012.

Esto significa que la suma del desinterés de política hídrica por el territorio y su condición de espacio fronterizo en disputa ha impedido que, por un lado, Guatemala desarrolle cierto interés por esta cuenca (que además es inexistente para los actores de la política hídrica de aquel país, como lo evidencian las entrevistas realizadas), y que, por el otro, exista una visión de cuenca a escala trinacional.

Así, si bien el diagnóstico financiado por la Conagua (2007) menciona que se trata de una cuenca trinacional y que Guatemala ocupa la parte más alta, toda la información se centra en México y Belice; los créditos a las estructuras gubernamentales se restringen a estos dos países. No hay datos de alguna temática procedentes de Guatemala y es menester señalar que también, en materia de GIRH y de aspectos de políticas hídricas, la parte mexicana del diagnóstico es más desarrollada, lo cual significa un desbalance con Belice. De igual forma, las 
recomendaciones derivadas del diagnóstico tienden a ignorar las problemáticas beliceñas de la cuenca.

Por lo tanto, existe un desequilibrio histórico en esta cuenca trinacional, cuya dinámica ha sido esencialmente binacional; a partir de esta característica, hay una fuerte contradicción en materia de GIRH, a causa de la exclusión de la parte alta de la cuenca de cualquier acción de gestión transfronteriza, situación que entra en total contradicción con la cuenca, en tanto territorio predilecto de expresión de este pilar de política mexicana del agua. Lo anterior constituye un elemento clave de inconsistencia en materia de gestión integrada de recursos hídricos.

En definitiva, con la instalación de la Comisión de Cuenca del Río Hondo (CCRH) en 2009, la dimensión de cuenca tiende a erosionarse aún más, en la medida en que ésta solamente abarca una fracción de la parte mexicana. Esto deriva en un plan de gestión mexicano posterior, que debe lidiar con problemáticas transfronterizas de contaminación de plagas, como el pez diablo (Hypostomus plecostomus), el cual, según las entrevistas realizadas con especialistas, presenta un indudable trasfondo transfronterizo, pues dicho pez ha cruzado varias fronteras antes de llegar a la parte mexicana del río Hondo; por ello, la solución también debería ser transfronteriza.

En consecuencia, desde hace una década existe una comisión de cuenca mexicana con cierto interés en acciones binacionales (MéxicoBelice) para una cuenca trinacional, situación que contraviene los principios básicos de la GIRH y su espacio predilecto de acción: la cuenca en la totalidad de su extensión.

En términos generales, esto constituye otra inconsistencia en materia de GIRH. Las entrevistas con actores vinculados con la CCRH 
evidencian una visión de la cuenca, esencialmente binacional, que evoca límites poco claros. A la lejana y muchas veces ignorada Guatemala, se suma el total olvido que también existe en México del estado de Campeche, pues presenta una porción territorial significativa de la cuenca, y que ambas entidades (Guatemala y Campeche) son fundamentales para entender la dinámica de la cuenca en su totalidad, ya que presentan la característica en común de ubicarse en la parte alta. Es por ello que el estado de Campeche figura como el gran ausente de la $\mathrm{CCRH}$, pues no sesiona, porque se representa únicamente la parte baja de la cuenca, es decir, sólo aquellos que se encuentran en el estado de Quintana Roo.

Además, es fundamental subrayar que, en la mayoría de las entrevistas, el discurso tiende a separar tres medios relacionados, aunque presentan una conectividad ambiental en la cuenca: el río Hondo, la Bahía de Chetumal donde desemboca y la parte marina. Los actores expresan una visión estrecha de las relaciones, esto es, poco integral y no integradora, además de circunscribir la cuenca a la corriente del río Hondo y no mirar a este territorio más allá de su corriente principal.

Tales elementos contradicen los preceptos de la GIRH y plantean una serie de limitantes históricos que no facilitan una visión amplia de cuenca para el río Hondo, sino que propagan una perspectiva fragmentada, determinada por los límites político-administrativos internacionales y estatales en México, y que se circunscribe al ámbito inmediato de acción de los actores entrevistados, es decir, a una visión de cuenca totalmente truncada, como la integración de la propia CCRH lo evidencia. Lo anterior constituye una inconsistencia existente desde la formación de la $\mathrm{CCRH}$, que ha perdurado a lo largo de los años. 


\section{Una suma de inconsistencias: ¿política hídrica con GIRH o versus GIRH?}

En la parte mexicana de la cuenca del río Hondo, la CCRH representa la expresión formal de la GIRH en la medida en que integra la estructura propuesta por la Conagua para su concreción en el área. Cabe señalar que cuando se abordó la problemática de la cuenca en los años 20142016, la CCRH apareció mencionada como central para este territorio y articuladora de distintos actores (véase Kauffer, manuscrito aceptado, s.f.). Así, se trata de un espacio de colaboración caracterizado por un fuerte componente académico, reconocido por su papel de información y difusión de los problemas de la cuenca como espacio de convergencia de actores.

Sin embargo, en paralelo con el reconocimiento de la presencia de un órgano encargado de la cuenca, subsiste poca claridad del tipo de órgano: ¿se trata de un consejo, una comisión, un comité? Los actores entrevistados, principalmente varios participantes en la propia $\mathrm{CCRH}$, desconocen su escala de acción y expresan confusión en torno a su calidad de "órgano auxiliar" del CCPY, en ocasiones Ilamándola consejo o incluso comité.

Dicha situación puede explicarse por la ignorancia en la problemática del río Hondo durante mucho tiempo por el propio CCPY, y 
Tecnología y

Ciencias $₫$ Agua
2020, Instituto Mexicano de Tecnología del Agua

Open Access bajo la licencia CC BY-NC-SA 4.0

(https://creativecommons.org/licenses/by-nc-sa/4.0/)

en las pocas relaciones de la comisión con su "hermano mayor" o, por lo menos, a través de vínculos poco claros y explícitos para los actores locales. En otros términos, esto es la consecuencia directa de una GIRH desvinculada de las realidades locales y organizada en torno a una verticalidad impulsada desde el centro de la república, controlada a través de dos mecanismos: la ausencia de figura jurídica propia de las organizaciones de cuenca en México resultante de la propia LAN y la dificultad para convertirse en instancia decisoria para la cuenca. Todo esto es resultado de los términos en los que la LAN estableció las organizaciones de cuenca a pesar de las reformas de 2004, que ampliaron la participación de los actores locales; difícilmente, estas instancias participativas se han convertido en verdaderos espacios de negociación y de toma de decisión en relación con las problemáticas de las cuencas. Existen excepciones de organizaciones que, a lo largo del tiempo, han podido lograr cierta autonomía jurídica o financiera, pero son contadas en el país, y su condición se debe a la presencia en su seno de ciertos liderazgos no gubernamentales o de élites político-económicas.

La herencia de una política centralizada se expresa de forma muy contundente por los actores entrevistados, quienes mencionan que el papel de la CCRH consiste, esencialmente, en proveer información a ciertos sectores, como se puede observar en otros casos (Kauffer, 2005) y, por lo tanto, no decide, actúa ni constituye una instancia de gestión del agua. No obstante, se reconoce su existencia para construir alianzas y establecer una coordinación entre distintos actores cuando han surgido problemáticas agudas, incluso con el sectorempresarial, por ejemplo, con la plaga de peces diablos, la cual será retomada más adelante: 
"Las fortalezas son su pluralidad en cuanto a los sectores... a ratos siento que están un poco sobrerrepresentados los empresarios, pero bueno (...) y su gran limitante, decíamos, es la falta de construcción internacional; tendría que haber alguien de Belice; si me apuras, hasta alguien de Guatemala". Entrevista con académico especialista de la cuenca, Chetumal, 21 de septiembre de 2016.

El segundo elemento relacionado con la política hídrica mexicana que ha llevado a una dinámica de inconsistencia de la CCRH ha sido la crisis de falta de recursos. Entre 2009 y 2015, mediante un proceso lento y paulatino de consolidación, la CCRH presentaba una perspectiva prometedora en materia de GIRH para gran parte de la región y la posibilidad de pensar alguna dimensión transfronteriza, donde la cooperación es una dimensión casi ausente (García \& Kauffer, 2011; Kauffer, 2017; Kauffer, 2018). Sin embargo, la falta de sostenibilidad a corto plazo de la CCRH fue puesta en evidencia a partir de 2016, cuando la disminución del financiamiento de la Conagua no solamente propició la imposibilidad de seguir con proyectos de investigación, sino casi la desaparición de la instancia, a su vez imposibilitada para realizar reuniones a medida que la figura del gerente no fuera reemplazada durante varios meses:

"A ese drama hemos llegado; te digo, habían funcionado los órganos porque había recurso federal para mantenerlos; pero una vez que eso no haya, yo creo que ya no van a funcionar; 
esa va a ser la parte... A la mejor el problema económico va a ser... pero ese... el drama principal (...).

Fue en abril la última sesión, 19 de abril de 2016, y ya no estaba viniendo XXX (nombre del gerente); de hecho, XXX creo que estaba presentando unos exámenes para ahuecar, porque ya no iba a tener posibilidades; fue la última reunión en abril y de ahí ya no tenemos gerente operativo.

(...) Y ahí nos reunimos los que pudimos, porque ya no hubo los recursos para unir físicamente a las personas y de entonces para acá estamos en punto muerto, porque yo creo que también se está esperando el cambio de gobierno". Entrevista con académica integrante de la CCRH, Chetumal, 20 de septiembre de 2016.

Así, en tercer lugar, la problemática de la dependencia de la CCRH de algunas personas (gerente 0 algunos investigadores de instituciones académicas) es decir, un tipo de GIRH personalizado y alejado del paradigma del espacio participativo que pretende constituirla perspectiva de la GIRH construida en México o, por lo menos, tal como aparece presentada en los documentos, es una realidad muy patente a escala local.

Cabe agregar una vulnerabilidad a los cambios de gobierno derivada del modelo establecido por el gobierno federal mexicano, el cual, en el sur de México, después de controlar directamente las organizaciones de cuenca a través de la Conagua, ha delegado ciertas funciones a escala local (municipal o estatal), situación que ha fragilizado estructuras no 
consolidadas aún en materia de GIRH debido a los cambios políticos trienales y sexenales.

En cuarto lugar, la inconsistencia principal de la GIRH en la CCRH está arraigada en las perspectivas de los actores en torno a la cuenca, que se han reflejado en la construcción de la propia comisión, solamente mexicana. Varios aspectos entran en juego para explicar tal situación, en primer lugar, la propia condición errática de la GIRH, aunque asumida como eje de una política del agua sectorial y fragmentada en Belice (Kauffer \& Mejía, 2020):

"Hasta ahorita nadie lo ha abarcado, en el país de Belice nos hace falta hacer algo como una comisión que tenga que ver con cuencas, entonces no está establecido, todos trabajan en lo que les pertenece". Entrevista con un funcionario del gobierno de Belice, Belmopán, 26 de marzo de 2007.

La dificultad de pensar en la dimensión transfronteriza de la cuenca del río Hondo es una realidad en ambos países, incluso cuando existe colaboración transfronteriza en otros aspectos:

"Desgraciadamente, ninguna actividad sobre el río Hondo, como la contaminación, está contemplada en el gobierno de México o en el de Belice. Cualquier actividad está contemplada sobre la construcción; el cruce del puente está contemplada en los propósitos para dar recomendaciones u otros. En este nivel no se ha hecho algo para evitar conflictos, no se ha contribuido en 
este sentido, pero se debería hacer algo". Entrevista con funcionario del gobierno de Belice, Belmopán, 28 de marzo 2007.

Finalmente, el tema de las asimetrías económicas y políticas entre los dos estados, que lleva a México a asumir la totalidad de los costos financieros y el liderazgo para la gestión de la cuenca, es una cuestión compleja en la medida en que los temas transfronterizos del agua en México no resultan de gran interés para su gobierno del sur (Kauffer, 2018), lo que conlleva a tensiones entre las propias instituciones del gobierno federal.

"¿Qué acuerdo hicimos con la situación de Belice? ¿Cómo está el recurso? Esto lo sabíamos desde antes, desde hace mucho tiempo, que es un país que no tiene recursos, que no tienen asistencia, por eso ofrecimos, como gobierno de México, hacer nosotros la iniciativa, fue de nosotros, de México; los términos de referencia del estudio salieron de nosotros y nosotros ofrecimos hacerlo en los dos países, para tener esa información, conscientes de que ellos no tenían los recursos; no era un estudio que se iba hacer una parte y la contraparte beliceña, no, no confundamos eso, por favor. El estudio fue hacerlo nosotros, las dos partes. Pactamos en él, ese fue el compromiso formal que adquirimos, nunca nos pidieron, nosotros lo ofrecimos a los beliceños y lo ofrecimos en esos términos, esa es la realidad. Así de simple los costos, así fue; si nos equivocamos en el 2003 al haberlo ofrecido cuando no podíamos hacerlo, entonces fue un 
error garrafal que tuvimos en esa época. Pero fue un ofrecimiento nuestro a los beliceños y no es que esté defendiendo la intervención de los beliceños, estoy defendiendo el compromiso internacional que asumimos y que adquirió nuestro gobierno mexicano". Funcionario del gobierno federal mexicano, Segunda Reunión del Consejo de Cuenca de la Península de Yucatán, Grupo de Trabajo Especializado en Saneamiento de Quintana Roo (GTESQROO), Chetumal, México, 24 de agosto de 2006.

Así, las consideraciones políticas y económicas nacionales e internacionales son imprescindibles para entender la suma de inconsistencias observadas, en particular en la CCRH, la cual, si bien es el principal instrumento de la GIRH en la cuenca del río Hondo, refleja una serie de contradicciones nacionales e internacionales de las políticas hídricas de los tres países que conforman este escenario transfronterizo.

\section{GIRH y SH en la cuenca del río Hondo: una triple relación}

En el contexto antes mencionado, caracterizado por una suma de inconsistencias históricas y actuales en torno a la GIRH, la cuenca del río Hondo evidencia tres modalidades de interacciones entre GIRH y $\mathrm{SH}$ 
desde la amplia perspectiva de las seguridades-inseguridades hídricas construidas y vividas: la ignorancia de la noción actual de seguridad hídrica por los actores de la GIRH que participan en la $\mathrm{CCRH}$; la persistencia de antiguas problemáticas hídricas que se convierten en escenarios de inseguridades hídricas para distintos actores locales, y cómo en condiciones agudas, éstas contribuyen a la SH a partir de la existencia de la plataforma de la CCRH y su orientación hacia la gestión integrada de recursos hídricos.

\section{Una seguridad hídrica ignorada y desvinculada de la GIRH}

En la cuenca del río Hondo, el tema de la seguridad hídrica es casi ignorado por los actores, que difícilmente logran identificar la problemática (sólo 3 de 13 entrevistados en 2016 lo hicieron) sin poder precisar las acciones de la $\mathrm{CCRH}$ en materia de $\mathrm{SH}$ en el territorio. En términos generales, los actores desconocen el concepto y cuando lo evocan, la visión de éste es parcial y no incluye los cuatro componentes antes mencionados: 1) la seguridad hídrica a través de indicadores tradicionales de escasez y de estrés hídrico; 2) la vulnerabilidad relacionada con sequías e inundaciones; 3 ) la seguridad hídrica enfocada hacia las dimensiones humanas en materia de seguridad alimentaria, y 4) la SH asociada con sustentabilidad.

En el río Hondo, la seguridad hídrica, cuando es vista por los actores entrevistados, remite a tres aspectos: disponibilidad de agua, acceso a 
Tecnología y

Ciencias $\underset{\mho}{\mho}$ gua
2020, Instituto Mexicano de Tecnología del Agua Open Access bajo la licencia CC BY-NC-SA 4.0

(https://creativecommons.org/licenses/by-nc-sa/4.0/)

agua potable y problemática de la contaminación. A partir de estos elementos, si bien existe una preocupación por mejorar la calidad del agua, tema muy mencionado por los distintos actores, la SH no es vista con claridad y las acciones de la CCRH no están vinculadas con esta noción que no parece concretarse en las escalas locales. En algunos casos, la seguridad hídrica se menciona desde el punto de vista de la seguridad nacional debido a la ubicación del río Hondo en un territorio transfronterizo, donde la seguridad fronteriza ha sido reforzada en el lado mexicano con dos bases militares a lo largo del río, y con la presencia de elementos de la Marina y de otras corporaciones en algunas localidades ubicadas en sus colindancias.

Cabe señalar que en los objetivos de alineación del Plan Rector en Materia de Agua para la Protección, Conservación y Recuperación Ambiental de la Península de Yucatán para el periodo 2015-2024 (Conagua, 2015), la seguridad hídrica sólo queda enunciada en materia de prevención de sequías e inundaciones en cuatro problemáticas: reducción de la cantidad y calidad del agua, deterioro de los recursos naturales, fenómenos extremos y cambio climático. En los detalles de los ejes, objetivos y estrategias del Programa Hídrico Visión 2030 de la Península de Yucatán, y de los problemas prioritarios, objetivos estratégicos, metas generales y acciones, así como en el Plan Rector actualizado en el año 2015, las referencias a la seguridad hídrica desaparecen, lo cual significa que sólo quedan plasmadas a escala federal, sin presentar una implementación en la cuenca del río Hondo.

El hallazgo que se derivó a la par de las entrevistas y de los documentos institucionales evidenció que mientras a escala local la seguridad hídrica es poco evocada y conocida, cuando se menciona, se 
remite a disponibilidad, acceso y calidad del agua del lado mexicano de la cuenca; es decir, que no abarca la amplitud del concepto propuesto a escala internacional y por la propia Conagua. Por su parte, en la visión institucional predomina la temática de sequías e inundaciones. Ello significa que, en ningún momento, la GIRH y SH están claramente vinculadas ni para los actores locales relacionados con la CCRH (en tanto espacio predilecto de la GIRH formal) ni para aquellos relacionados con la GIRH a escala regional a través del CCPY, siendo el concepto de SH enunciado de manera exclusiva en los objetivos nacionales de la propia Conagua.

\section{Las inseguridades hídricas duraderas en el río Hondo}

Si se centra la reflexión en torno a las inseguridades hídricas vistas como producto de las relaciones de poder, es fundamental remitir a las grandes problemáticas de la cuenca del río Hondo que afectan a las poblaciones locales, sus ecosistemas y viceversa.

Del lado mexicano, históricamente, la principal fuente de inseguridades hídricas en la cuenca del río Hondo ha sido la contaminación de la corriente principal, resultante de una doble dinámica: el uso de agroquímicos en las actividades agrícolas y las deficiencias en el tratamiento de aguas residuales. El principal evento detonador reportado en el diagnóstico (Conagua, 2007) y por muchas personas entrevistadas fue una mortandad de peces ocurrida en 2006 en la bahía de Chetumal. 
Tecnología y

Ciencias $\stackrel{\mho}{\mho}$ gua
2020, Instituto Mexicano de Tecnología del Agua

Open Access bajo la licencia CC BY-NC-SA 4.0

(https://creativecommons.org/licenses/by-nc-sa/4.0/)

Los estudios realizados identificaron los agroquímicos, cuyo origen se relaciona con la fuerte actividad cañera en ambos márgenes del río, pero principalmente en la parte beliceña. Esta situación afecta la pesca de autoconsumo, que aunque no es comercial, es una de las principales actividades humanas registradas en el río Hondo en el trabajo de campo (a la par de los pasos transfronterizos de contrabando de mercancías). Así, la contaminación del río crea un problema sanitario que tiene efectos en la salud de la población y en las actividades recreativas, por ejemplo, el de desarrollo del turismo, sector emergente en el río Hondo.

En el diagnóstico (Conagua, 2007), una de las principales problemáticas de la cuenca que afecta a los pobladores aparece en la literatura internacional y en los documentos nacionales de la Conagua desde la perspectiva de una visión tradicional de SH. Se trata del tema de las inundaciones por la ocurrencia de huracanes, mencionada como un elemento que también puede considerarse desde una perspectiva más amplia y socio-política, como parte de las inseguridades hídricas para las poblaciones del río Hondo, principalmente en la parte beliceña. Así, en las entrevistas realizadas en Belice, se trata de la problemática más evocada para el río Hondo y del país. En Belice, las áreas más pobres se caracterizan por huellas duraderas de las inundaciones en sus construcciones y es notable la presencia de albergues destinados a la protección de la población, situación que no se observa del lado mexicano, donde predomina la temática de contaminación del recurso hídrico. Dicha situación subraya que las inseguridades hídricas pueden percibirse de forma distinta por los actores que pueblan los márgenes de un mismo río, usan el recurso hídrico para diversas actividades o realizan acciones en materia de agua en una determinada cuenca. 
Una de las problemáticas mencionadas por los actores mexicanos es el proceso de deforestación de las partes medias de la cuenca que produce erosión, pérdida de suelo y escurrimiento de sedimentos a la corriente principal. El diagnóstico (Conagua, 2007) lo refiere con claridad y es un elemento reiterativo de las entrevistas realizadas. Ello repercute en la Reserva de la Bahía de Chetumal, área natural estatal protegida, santuario del manatí, una especie en peligro de extinción. A medida que se trata de la parte baja de la cuenca, es el lugar donde se cosechan las consecuencias de todas las acciones y actividades llevadas a cabo en las partes altas y medias de la misma.

El acceso a agua de calidad para la población es un tema importante en la cuenca del lado mexicano. En la parte de Calakmul, Campeche, poco visualizada en la cuenca, no existen aguas superficiales y las subterráneas presentan problemas de dureza (como es el caso del propio río Hondo), que impiden su consumo humano y, por lo tanto, el acceso de la población a agua para uso doméstico proveniente del río.

El tema del saneamiento sigue siendo una dificultad, pues alrededor de $50 \%$ de las aguas se tratan en la parte mexicana de la cuenca, lo que repercute en un ambiente sano, en cuestión de salud; pero pone en peligro las actividades realizadas en sistemas lagunares asociados, como las lagunas de Bacalar, conectadas de modo superficial y subterráneo con el río Hondo.

De este modo, las antiguas problemáticas no resueltas de la cuenca del río Hondo expresan una serie de inseguridades hídricas para los pobladores de ese territorio, e impactan de forma diferenciada en función de su localización; pero también según las actividades que desarrollan y su grado de exposición, sobre todo cuando viven en condiciones de 
marginación, situación particularmente evidenciada durante los recorridos fluviales realizados en la frontera beliceña. Esto significa que las problemáticas del agua perduraron con la GIRH y que a las antiguas se sumaron nuevas inseguridades hídricas en la cuenca.

\section{Inseguridades hídricas agudas: la CCRH, plataforma de la GIRH}

En los últimos años, el pez diablo ha sido uno de los más agudos problemas ambientales que se presentaron en la cuenca del río Hondo. Primero, el pez fue detectado en Guatemala, en la cuenca del río Usumacinta; después en la parte alta del río Hondo, donde escurre el Arroyo Azul; posteriormente en Belice y, por último, en la parte mexicana de la cuenca. Su presencia afecta al ecosistema ribereño a medida que el pez anida en las riberas y provoca erosiones en sus márgenes, y representa un peligro para otras especies de peces porque come sus huevos; por ende, afecta el equilibro de los sistemas acuáticos y las actividades pesqueras. Parte de la peligrosidad de esta especie radica en su llegada a las lagunas de Bacalar, al cambiar las dinámicas de los ecosistemas y quizás por la coloración de las lagunas (según los especialistas entrevistados), que representan el principal atractivo del área, lo que conlleva efectos económicos de amplitud para el turismo. No obstante, en fechas recientes, el impacto de esta especie invasora ha sido calificado como estable, a diferencia de otros ríos en el país (Maldonado, 2019). 
Lo interesante acerca del pez diablo fue que su presencia generó una situación de inseguridad hídrica concreta, aguda y de emergencia, y que ciertos actores relacionados con la CCRH pudieron dar una respuesta sin contar con el apoyo de la Conagua. El siguiente extracto de entrevista lo evidencia con claridad:

"X... sabía que yo formaba parte de ese órgano auxiliar del río Hondo, y viene y me plática: 'oye, Z..., fíjate que un investigador de Belice me dijo que ya encontraron pez diablo en un tributario que desemboca al río Hondo'. Z... ya empecé a saber qué era el pez diablo y su impacto, y lo que tenía: '¿Entonces por qué no se busca hacer algo dentro de ese comité (sic)?'. Bueno, entonces yo, como parte de ese comité, hago una invitación y pido que se haga una reunión para que podamos avanzar con eso... Cuando yo voy a la instancia, Conagua, es quien dirige más arriba esto, entonces yo les digo: 'Oye, inviten a una reunión, convoquen a todos los miembros, porque está esta situación y es delicada, y es grave y puede pasar esto y esto' [...] 'Sí, Z..., pero es que ahorita estamos atendiendo no sé qué bomberazos institucionales de la Conagua'. Entonces, como que ellos estaban con su prioridad, entonces yo dije: 'bueno, está grave'. Entonces, ¿qué fue lo que hice? Agarré la lista de distribución de todos los miembros del comité de cuenca (sic) del río Hondo y les platico [...] Entonces, sorprendentemente, los que me respondieron inmediatamente buscando apoyar de la manera que fuera fueron los empresarios, los empresarios que formaban parte de ese comité de cuenca, no las instituciones". 
Entrevista con miembro del sector académico, CCRH, Chetumal, 20 se septiembre de 2016.

De esta manera, si bien existe una continuidad e incluso, con la aparición del pez diablo, un aumento de las situaciones de inseguridades hídricas en la cuenca del río Hondo, y a pesar de que la noción de seguridad hídrica no aparezca claramente en el discurso de los actores locales ni en los documentos en materia de GIRH en la región, en los hechos -el ejemplo del pez diablo como elemento de inseguridad hídrica aguda, tal como lo fue, en su momento, el tema de la contaminación del río- ha logrado conjuntar esfuerzos de diversos actores organizados en torno a la CCRH; esto permite indicar que, en los hechos, las inseguridades hídricas concretas pueden convertirse en motores de acciones de la GIRH en esta escala, aunque los actores desconozcan la noción propuesta por las instancias internacionales y las discusiones desarrolladas por los académicos. Así, ante la situación aguda, la CCRH se convirtió en una plataforma que permitió articular una respuesta frente a la aparición del pez diablo: la CCRH funcionó como espacio de interacción entre actores, y permitió establecer un vínculo entre la GIRH y la $\mathrm{SH}$, a raíz de una situación de inseguridad hídrica aguda.

Las relaciones entre GIRH y $\mathrm{SH}$, caracterizadas por una falta de articulación en la cuenca del río Hondo presentan una versión desfavorable (GIRH combinada con inseguridades hídricas), pero además una modalidad donde la GIRH sirve de herramienta (vía la $\mathrm{CCRH}$ ) para impulsar una respuesta concreta ante una situación aguda de inseguridad hídrica. 
Por lo tanto, se ha podido identificar una triple modalidad de interacciones entre GIRH y SH en la cuenca del río Hondo a partir del análisis de las perspectivas de los actores involucrados en la $\mathrm{CCRH}$, de la predominante desvinculación entre ambas y de la relación inversa (GIRH con inseguridades hídricas acentuadas); en ocasiones se produce una interacción positiva, donde la GIRH permite una respuesta colaborativa entre actores frente a una situación de inseguridad aguda en la cuenca.

\section{Conclusiones}

En el sur de México, y en el caso particular de la cuenca del río Hondo, las acciones en relación con los principios de la GIRH y de la $\mathrm{SH}$ se caracterizan por un desfase temporal con respecto a los planteamientos internacionales e incluso nacionales. La GIRH que aparece en 1992 a escala internacional, como un principio de política hídrica se concreta en 1999-2000 a escala nacional, y toma forma hasta 2009, con la creación de la Comisión de Cuenca del Río Hondo (CCRH) en esta subcuenca. Por su parte, la SH acuñada en el año 2000 y aparecida en el PNH de la Conagua hasta 2014, continúa sin visibilidad clara en el río Hondo, bajo la perspectiva de los actores locales asociados con la Comisión de Cuenca del Río Hondo. 
A pesar de contar con un instrumento formal a través de la CCRH desde el año 2009, las inconsistencias entre los planteamientos de la GIRH a escala nacional, su marco jurídico, implementación e inadecuaciones a una cuenca transfronteriza (compartida físicamente, pero no desde una perspectiva político-institucional), se han sumado al reciente repliegue económico del gobierno federal mexicano para financiar las estructuras creadas en materia de gestión integrada de recursos hídricos.

Por otro lado, existe una clara desarticulación de la SH con la GIRH, situación que fue evidenciada desde la escala nacional, donde no se relacionan en lo jurídico ni en la política hídrica al interior de las instituciones creadas bajo el contexto de la GIRH. En el caso del río Hondo, el concepto de SH se revela ausente, desconocido y reducido a una mínima expresión entre los actores locales relacionados con la GIRH, en un contexto donde, por motivos históricos y debido a las transformaciones recientes en materia de política nacional, esta evidencia una suma de inconsistencias. En tal escenario, la persistencia de problemáticas existentes en materia de GIRH y la aparición de nuevas situaciones vinculados con el estado de los recursos hídricos plantean verdaderas inseguridades hídricas para la población local, las cuales se expresan de manera diferenciada según los actores y el país. En este caso, la relación entre los dos pilares de la política mexicana (GIRH y $\mathrm{SH}$ ) presentan una expresión inversa, donde la GIRH coexiste con una serie de inseguridades hídricas. No obstante, como lo evidencia la situación del pez diablo, las inseguridades hídricas permiten establecer puentes entre la $\mathrm{SH}$ y la GIRH, y de alguna forma, aprovechar la plataforma existente de interacciones en el seno de la $\mathrm{CCRH}$, para que cuando surjan de forma 
aguda inseguridades hídricas puedan, mediante una respuesta adecuada, contribuir al fortalecimiento de la GIRH, al facilitarla colaboración entre los actores locales que atienden la SH sin nombrarla, y aunque tampoco se propongan claramente tal objetivo.

\section{Agradecimientos}

A la Convocatoria de Problemas Nacionales (PDCPN) 2014 del Conacyt para el financiamiento del proyecto 248954.

\section{Referencias}

Alija, A. M. (2019). La situación actual de los conflictos territoriales y fronterizos en América Latina y Caribe. Journal of International Relations, (15), 1-15. DOI: 10.14422/cir.i15.y2019.001

Benítez, J. A. (2010). Situación actual de las cuencas de los ríos Candelaria y Hondo. En: Cotler, H. (ed.). Las cuencas hidrográficas de México. Diagnóstico y priorización (pp. 203-209). Recuperado de https://micrositios.inecc.gob.mx/publicaciones/libros/639/rcandela ria.pdf

Biswas, A. K. (2004). Integrated water resources management: a reassessment: A water forum contribution. Water International, 29(2), 248-256.

Biswas, A. K. (2008). Current directions: Integrated water resources management-a second look. Water International, 33(3), 274-278.

CCGSS, Centro del Cambio Global y la Sustentabilidad en el Sureste. (2015). Proyecto FORDECYT-Usumacinta. Cambio global y 
Ciencias $\approx$ Agua

sustentabilidad en la cuenca del río Usumacinta y zona marina de influencia. Bases para la adaptación al cambio climático desde la ciencia y la gestión del territorio. Recuperado de https://www.usumacinta.org

Conagua, Comisión Nacional del Agua. (2007). Diagnóstico para el manejo sustentable del agua en la cuenca Internacional del río Hondo México-Belice al 2025. México, DF, México: Comisión Nacional del Agua.

Conagua, Comisión Nacional del Agua. (2014). Programa Nacional Hídrico 2014-2018. México DF, México: Comisión Nacional del Agua.

Conagua, Comisión Nacional del Agua. (2015). Plan rector en materia de agua para la protección, conservación y recuperación ambiental de la Península de Yucatán, México. México DF, México: Comisión Nacional del Agua.

Conagua, Comisión Nacional del Agua. (2016). Estadísticas del Agua 2016. Ciudad de México, México: Comisión Nacional del Agua.

Cook, C., \& Bakker, K. (2012). Water security: Debating an emerging paradigm. Global Environmental Change, 22(1), 94-102.

García, G. A., \& Kauffer, M. E. F. (2011). Las cuencas compartidas entre México, Guatemala y Belice: un acercamiento a su delimitación y problemática general. Frontera Norte, 23(45), 131-161.

GWP, Global Water Partnership. (2000a). Manejo integrado de recursos hídricos. Stockholm, Sweden: Global Water Partnership.

GWP, Global Water Partnership. (2000b). Towards water security: A framework for action. Stockholm, Sweden: Global Water Partnership. 
Hidalgo, J. P., Boelens, R., \& Vos, J. (2017). De-colonizing water. Dispossession, water insecurity, and Indigenous claims for resources, authority, and territory. Water History, 9(1), 67-85.

INBO, GWP, UNECE, GEF, UNESCO, \& AFDE, International Network of Basin Organizations; Global Water Partnership; United Nations Economic Commission for Europe; Global Environmental Facility; United Nations Educational, Scientific and Cultural Organization, \& Agence Française de Développement. (2012). Handbook for Integrated Water Resources Management in Transboundary Basins of Rivers, Lakes and Aquifers. Paris, France: International Network of Basin Organizations (INBO), Global Water Partnership (GWP), United Nations Economic Commission for Europe (UNECE), Global Environmental Facility (GEF), United Nations Educational, Scientific and Cultural Organization (UNESCO), Agence Française de Développement (AFDE).

Kauffer, E., \& Gallardo, V. A. (2019a). Policy Brief 1. GIRH-SH y legislación en México. Lecciones a partir de ocho estudios de caso para una gestión local integral de recursos hídricos (GLIRH) frente a una gestión insegura estatal de recursos hídricos (GIERH) con seguridad hídrica (SH). Ciudad de México, México: Centro de Investigación y Estudios Superiores en Antropología Social (CIESAS), Instituto de Ecología (INECOL), Colegio de San Luis (COLSAN).

Kauffer, E. (2005). El Consejo de Cuenca de los ríos Usumacinta y Grijalva: los retos para concretar la participación y la perspectiva de cuencas. En: Vargas, S., \& Mollard, E. (eds.). Problemas socioambientales y experiencias organizativas en las cuencas de méxico 
Ciencias $\approx$ Agua

(pp. 195-218). México, DF, México: Instituto Mexicano de Tecnología del Agua (IMTA), Institut de Recherche pour le Développement.

Kauffer, E. F. (2014). Introducción. De las cuencas a la gestión integrada de recursos hídricos en Chiapas: una construcción histórica plasmada en escenarios concretos. En: Kauffer, M. E. F. (ed.). Cuencas en Chiapas: la construcción de utopías en cascada (pp. 1751). México, DF, México: Centro de Investigación y Estudios Superiores en Antropología Social (CIESAS).

Kauffer, E. (2017). Contrasting water securities: The Mexican state facing downstream stakeholders in the Suchiate transboundary river basin. International Journal of Water Resources Development, 35(1), 30-48. DOI: http://dx.doi.org/10.1080/07900627.2017.1393400

Kauffer, E. (2018). Un análisis de la gobernanza multinivel en las aguas transfronterizas de México con Guatemala y Belice. Frontera norte, 30(60), 31-56.

DOI: http://dx.doi.org/10.17428/rfn.v30i60.1114

Kauffer, E. (2019). Las dimensiones políticas del agua: miradas cruzadas en torno a aguas turbulentas. Ciudad de México, México: Centro de Investigación y Estudios Superiores en Antropología Social (CIESAS), Biblioteca del agua.

Kauffer, E. (manuscrito aceptado) (s.f.). De las redes de actores sociales a las redes de políticas públicas: la cuenca transfronteriza del río Hondo (México-Belice). En: Morales-Fajardo, M. E \& Cadena- 
Ciencias $\approx$ Agua

Inostroza C. (eds.). ¿Redes y/o gobernanza? Experiencias de colaboración entre actores. Ciudad de México, México: UNAM.

Kauffer, E., \& Mejía, L. (2020). De la descolonización a la gestión integrada de recursos hídricos (GIRH): la política del agua de Belice a través del tiempo (1981-2015). Gestión y Política Pública, 29(1), 37-66. DOI: http://dx.doi.org/10.29265/gypp.v29i1.656

Maldonado, J. (2019). Pez diablo no representa riesgo para el río Hondo: Ecosur. No ha progresado su invasión como lo ha hecho en otros ríos. Recuperado de https://www.lajornadamaya.mx/2019-0829/Pez-diablo-no-representa-riesgo-para-el-rio-Hondo--Ecosur

Maganda, C. (2019). Policy Brief 2. Desde la experiencia local: aportaciones de las realidades locales a los actores políticos e institucionales que promueven la GIRH y la SH en México y viceversa. Ciudad de México, México: Centro de Investigación y Estudios Superiores en Antropología Social (CIESAS), Instituto de Ecología (INECOL), Colegio de San Luis (COLSAN).

Mejía, L., \& Pliego E. (2019). Policy Brief 3. La gestión integrada de recursos hídricos (GIRH) y la seguridad hídrica (SH) contrastadas con ocho estudios de caso: conflictividades hídricas y actores sociales. Ciudad de México, México: Centro de Investigación y Estudios Superiores en Antropología Social (CIESAS), Instituto de Ecología (INECOL), Colegio de San Luis (COLSAN).

Martínez, P. F., Díaz-Delgado, C., \& Moeller, C. G. (2017). Seguridad hídrica en México. Ciudad de México, México: Academia Mexicana de Ingeniería. 
Molle, F. (2006). Planning and managing water resources at the riverbasin level: Emergence and evolution of a concept (Vol. 16). Colombo, Sri Lanka: International Water Management Institute (IWMI).

Norman, E. S., Dunn, G., Bakker, K., Allen, D. M., \& De Albuquerque, R. C. (2013). Water security assessment: Integrating governance and freshwater indicators. Water Resources Management, 27(2), 535551.

Olvera, D. N., Kauffer, E. F., Schmook, B., \& Huicochea, L. (2011). Factores de conflicto en la cooperación por el agua en cuencas compartidas: caso río Hondo (México-Guatemala-Belice). Estudios Fronterizos, 12(24), 103-134.

Warner, J., Wester, P., \& Bolding, A. (2008). Going with the flow: River basins as the natural units for water management? Water Policy, 10(S2), 121-13.

Wester, P., \& Warner, J. (2002). River basin management reconsidered. In: Turton, A., \& Henwood, R. (eds.). Hydropolitics in the developing world: A Southern African perspective (pp. 61-71). Pretoria, South Africa: South African Water Issues Research Unit and International Water Management Institute. 